\title{
Guajadial: An Unusual Meroterpenoid from Guava Leaves Psidium guajava
}

\author{
Xiao-Long Yang, ${ }^{\dagger} \S \quad$ Kun-Lung Hsieh, ${ }^{\ddagger}$ and Ji-Kai Liu ${ }^{*}{ }^{\dagger}$
}

State Key Laboratory of Phytochemistry and Plant Resources in

West China, Kunming Institute of Botany, Chinese Academy of

Sciences, Kunming 650204, P. R. China

Hsiehs Biotech. Co., Ltd., 1-3 Cao Ba Nha St., Dist. 1,

Hochiming City Vietnam

Graduate School of Chinese Academy of Sciences, Beijing

100039, P. R.

China

* Corresponding author. Phone: 0086-871-5216327. Fax: 0086-871-5150227.

e-mail: jkliu@mail.kib.ac.cn

† Kunming Institute of Botany.

$\ddagger$ Hsiehs Biotech. Co., Ltd.

${ }^{\S}$ Graduate School of Chinese Academy of Sciences 


\section{List of Supporting Information}

Figure S1. EI-MS spectrum of Guajadial (1)...............................S3

Figure S2. ${ }^{1} \mathrm{H}$ NMR spectrum of Guajadial (1, $\left.\mathbf{4 0 0} \mathbf{M H z}, \mathbf{C D C l}_{3}\right) \ldots \ldots \ldots \ldots \ldots . . . . . .53$

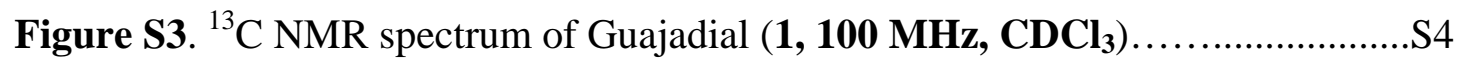

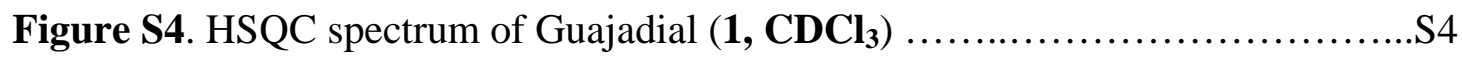

Figure S5. HMBC spectrum of Guajadial $\left(\mathbf{1}, \mathbf{C D C l}_{3}\right) \ldots \ldots \ldots \ldots \ldots \ldots \ldots \ldots \ldots \ldots$

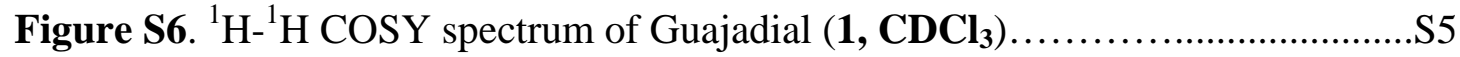

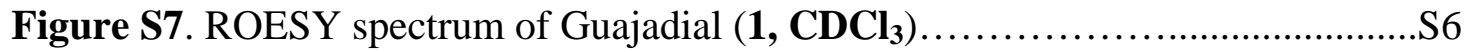

Figure S8. HRFAB-MS spectrum of Guajadial (1)............................

Figure S9. HRFAB-MS spectrum of fragment peak at $m / z 271$ of

Guajadial (1)................................................S8

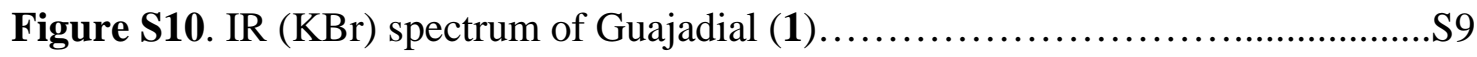

Figure S11. UV(MeOH) spectrum of Guajadial (1) $\ldots \ldots \ldots \ldots \ldots \ldots \ldots \ldots \ldots \ldots \ldots \ldots$. 9

Figure S12. ${ }^{1} \mathrm{H}$ NMR spectrum of Guajadial (1, $500 \mathbf{M H z}, \mathbf{C}_{5} \mathbf{D}_{5} \mathbf{N}$

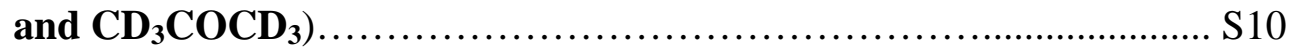

Figure S13. ${ }^{13} \mathrm{C}$ NMR spectrum of Guajadial $\left(\mathbf{1}, \mathbf{1 2 5} \mathbf{M H z}, \mathbf{C}_{5} \mathbf{D}_{5} \mathbf{N}\right.$

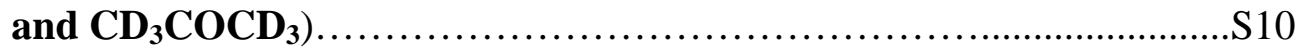

Figure S14. HSQC spectrum of Guajadial $\left(1, \mathbf{C}_{5} \mathbf{D}_{5} \mathbf{N}\right.$ and $\left.\mathrm{CD}_{3} \mathbf{C O C D}_{3}\right) \ldots \ldots \ldots \ldots \ldots . . . . . .11$

Figure S15. HMBC spectrum of Guajadial $\left(\mathbf{1}, \mathbf{C}_{5} \mathbf{D}_{5} \mathbf{N}\right.$ and $\left.\mathbf{C D}_{3} \mathbf{C O C D}_{3}\right) \ldots \ldots \ldots \ldots . . . . .11$

Figure S16. ${ }^{1} \mathrm{H}-{ }^{1} \mathrm{H}$ COSY spectrum of Guajadial $\left(\mathbf{1}, \mathbf{C}_{5} \mathbf{D}_{5} \mathbf{N}\right.$ and $\left.\mathbf{C D}_{3} \mathbf{C O C D}_{3}\right) \ldots \ldots . . . \mathrm{S} 12$

Figure S17. ROESY spectrum of Guajadial $\left(\mathbf{1}, \mathbf{C}_{5} \mathbf{D}_{5} \mathbf{N}\right.$ and $\left.\mathbf{C D}_{3} \mathbf{C O C D}_{3}\right) \ldots \ldots \ldots \ldots . . . . .212$ 
Figure S1. EI-MS spectrum of Guajadial (1)

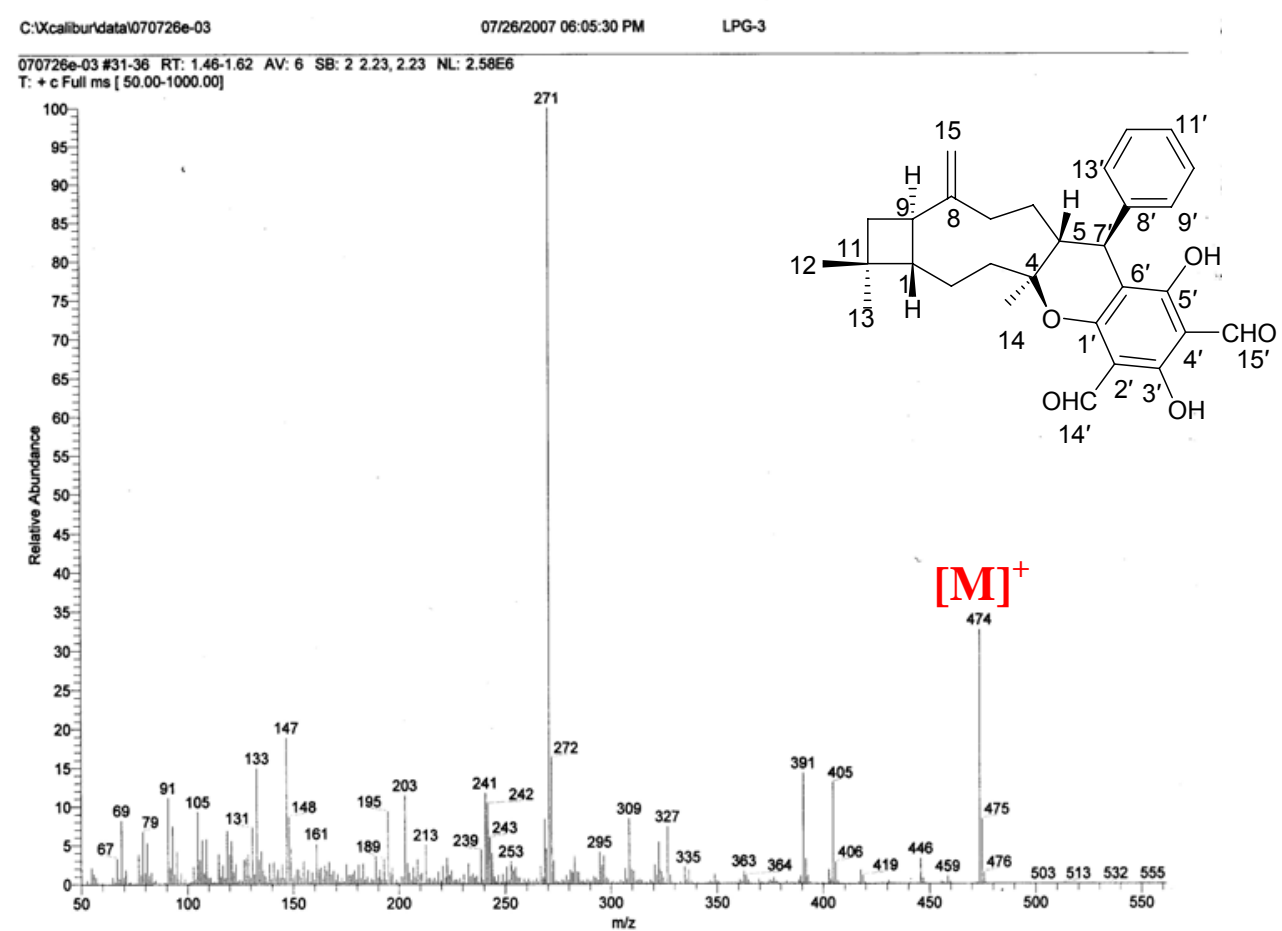

Figure S2. ${ }^{1} \mathrm{H}$ NMR spectrum of Guajadial $\left(\mathbf{1}, \mathbf{4 0 0} \mathbf{M H z}, \mathbf{C D C l}_{3}\right)$

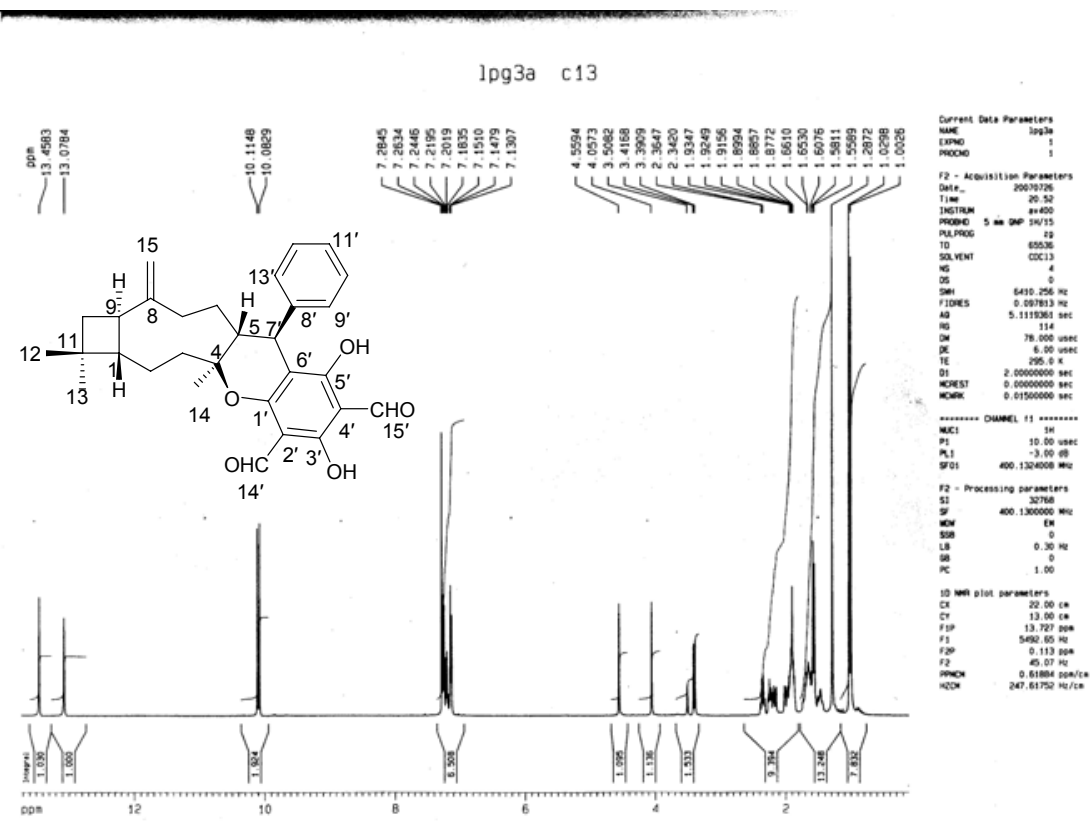


Figure S3. ${ }^{13} \mathrm{C}$ NMR spectrum of Guajadial $\left(\mathbf{1}, \mathbf{1 0 0} \mathbf{M H z}, \mathbf{C D C l}_{3}\right)$

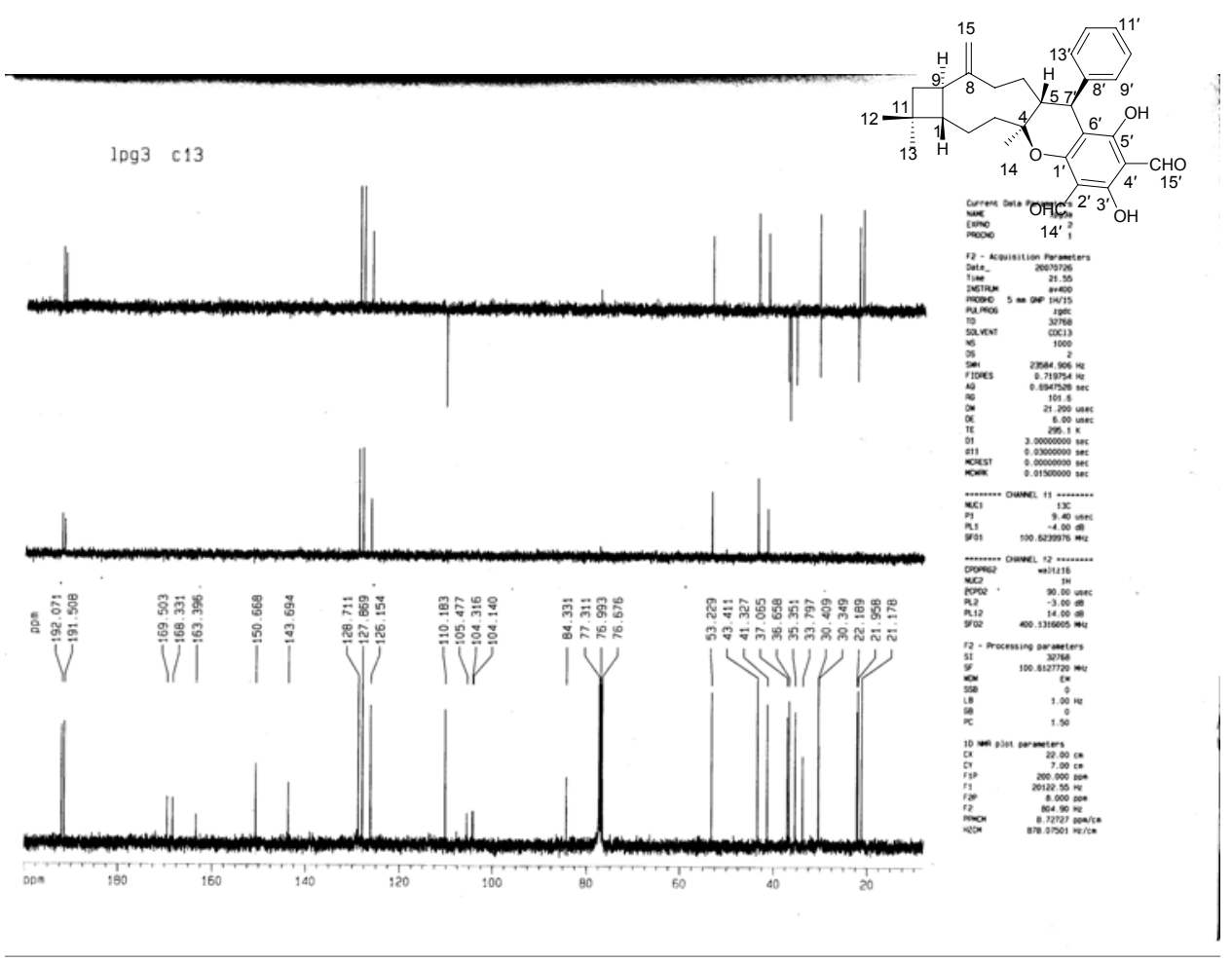

Figure S4. HMQC spectrum of Guajadial (1, $\left.\mathbf{C D C l}_{\mathbf{3}}\right)$

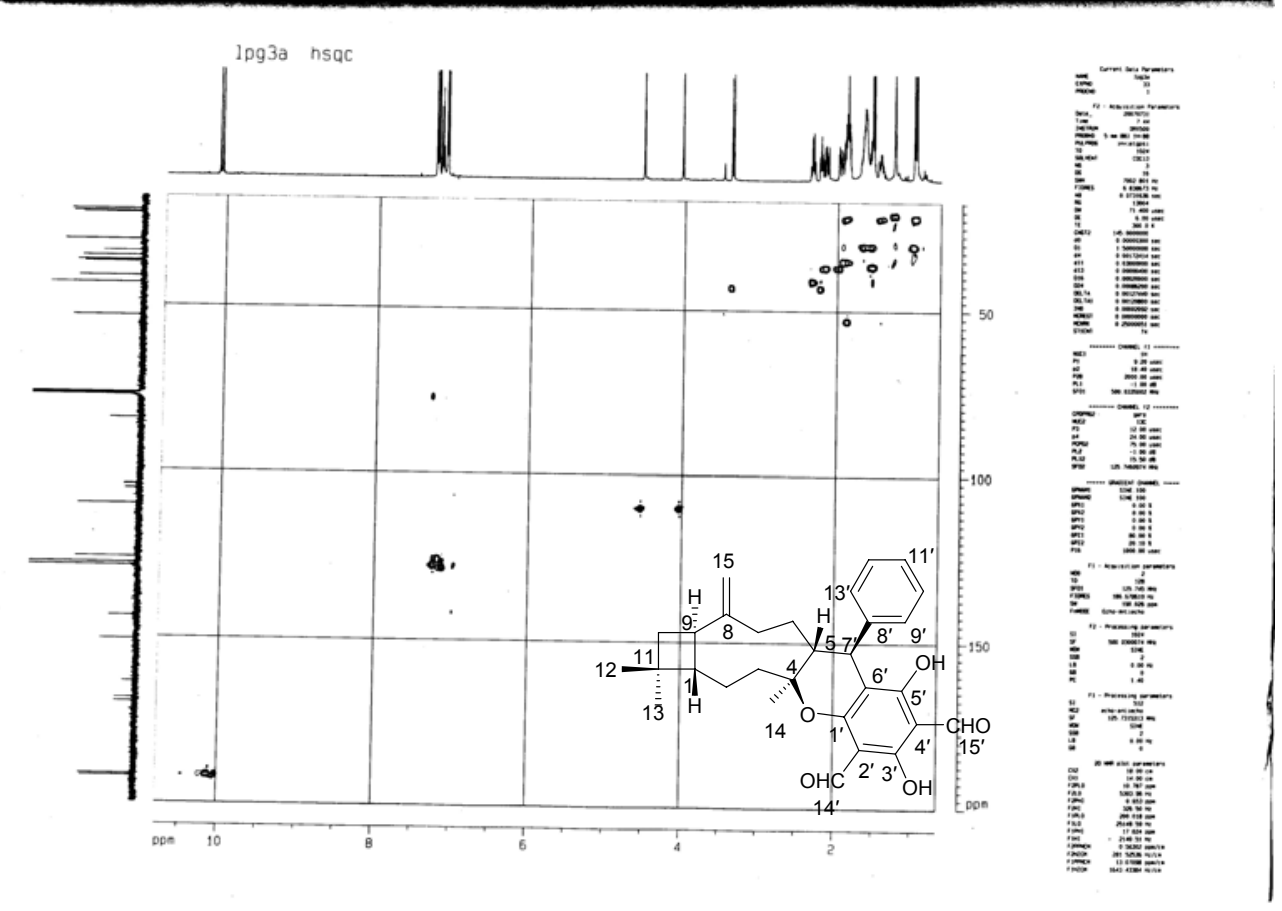


Figure S5. HMBC spectrum of Guajadial $\left(\mathbf{1}, \mathbf{C D C l}_{3}\right)$
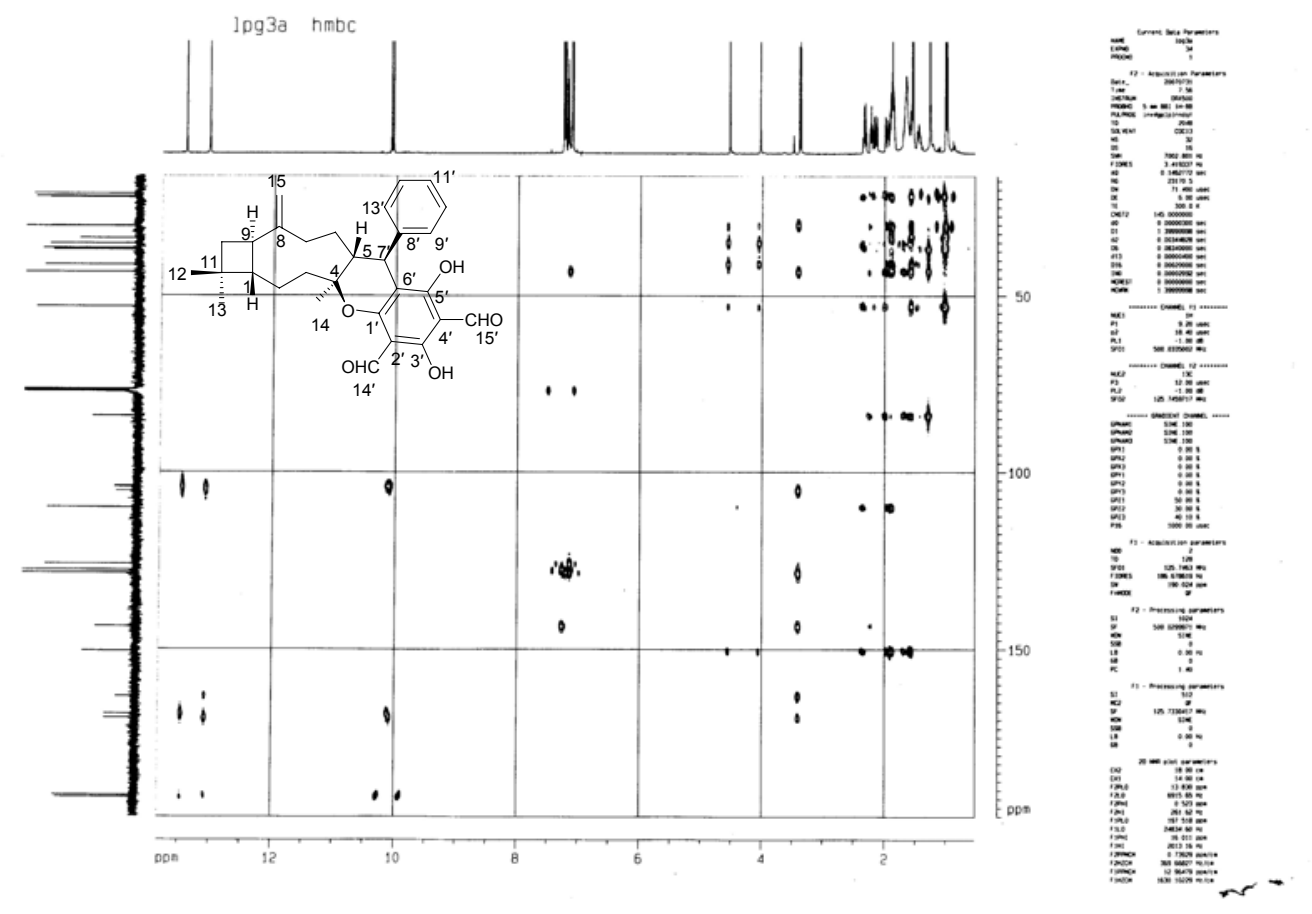

Figure S6. ${ }^{1} \mathrm{H}-{ }^{1} \mathrm{H}$ COSY spectrum of Guajadial (1, $\left.\mathbf{C D C l}_{3}\right)$

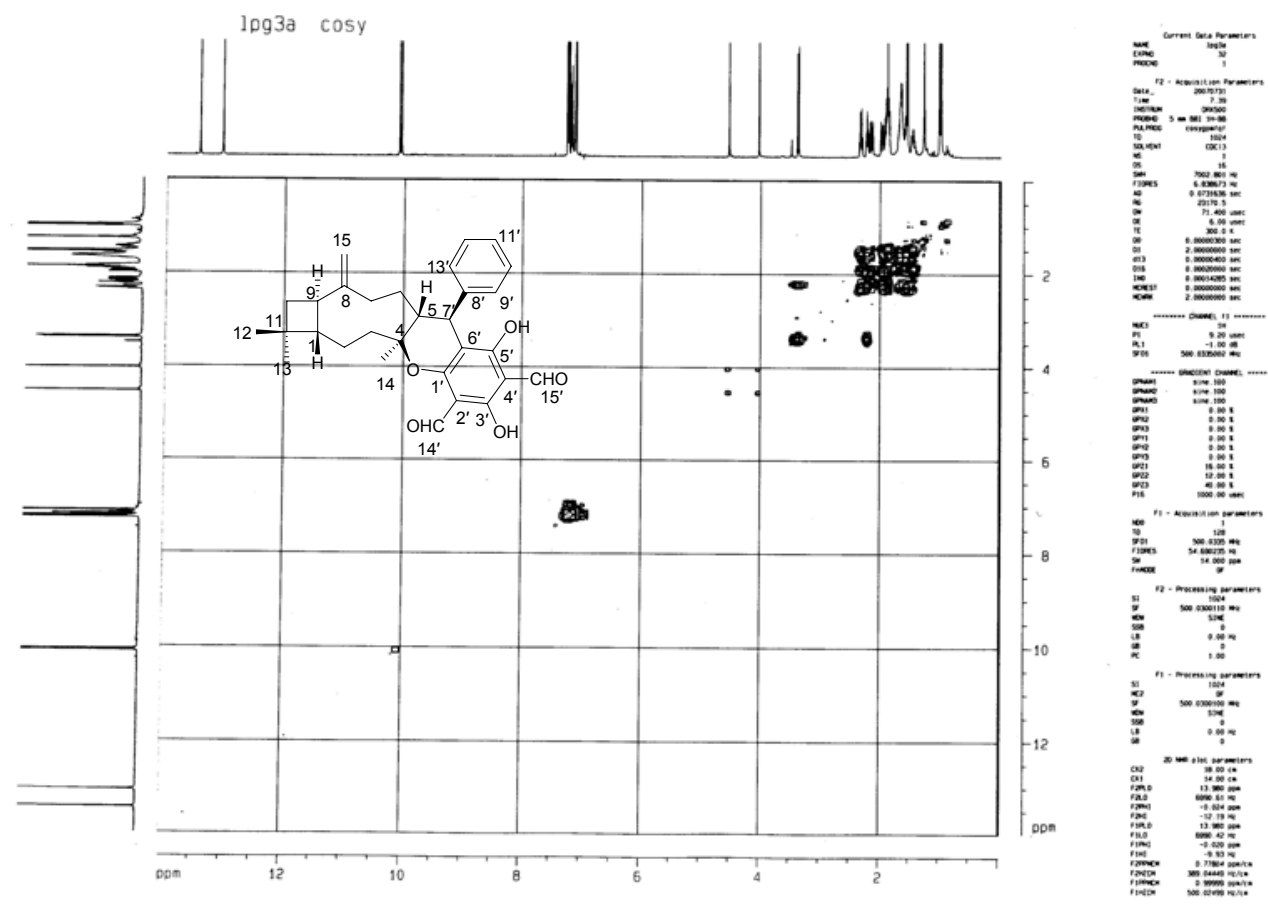


Figure S7. ROESY spectrum of Guajadial $\left(\mathbf{1}, \mathbf{C D C l}_{3}\right)$
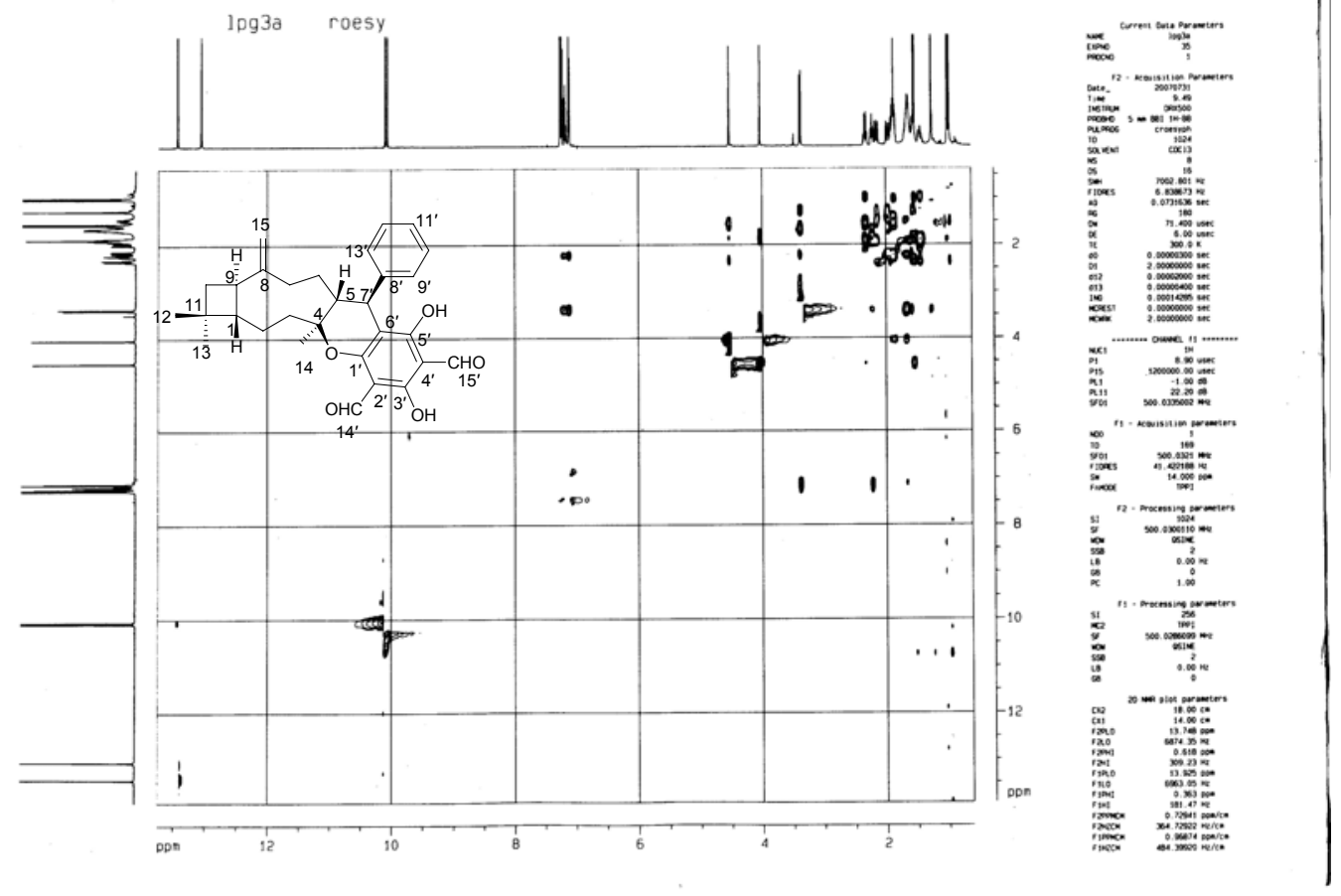
Figure S8. HRFAB-MS spectrum of Guajadial (1)
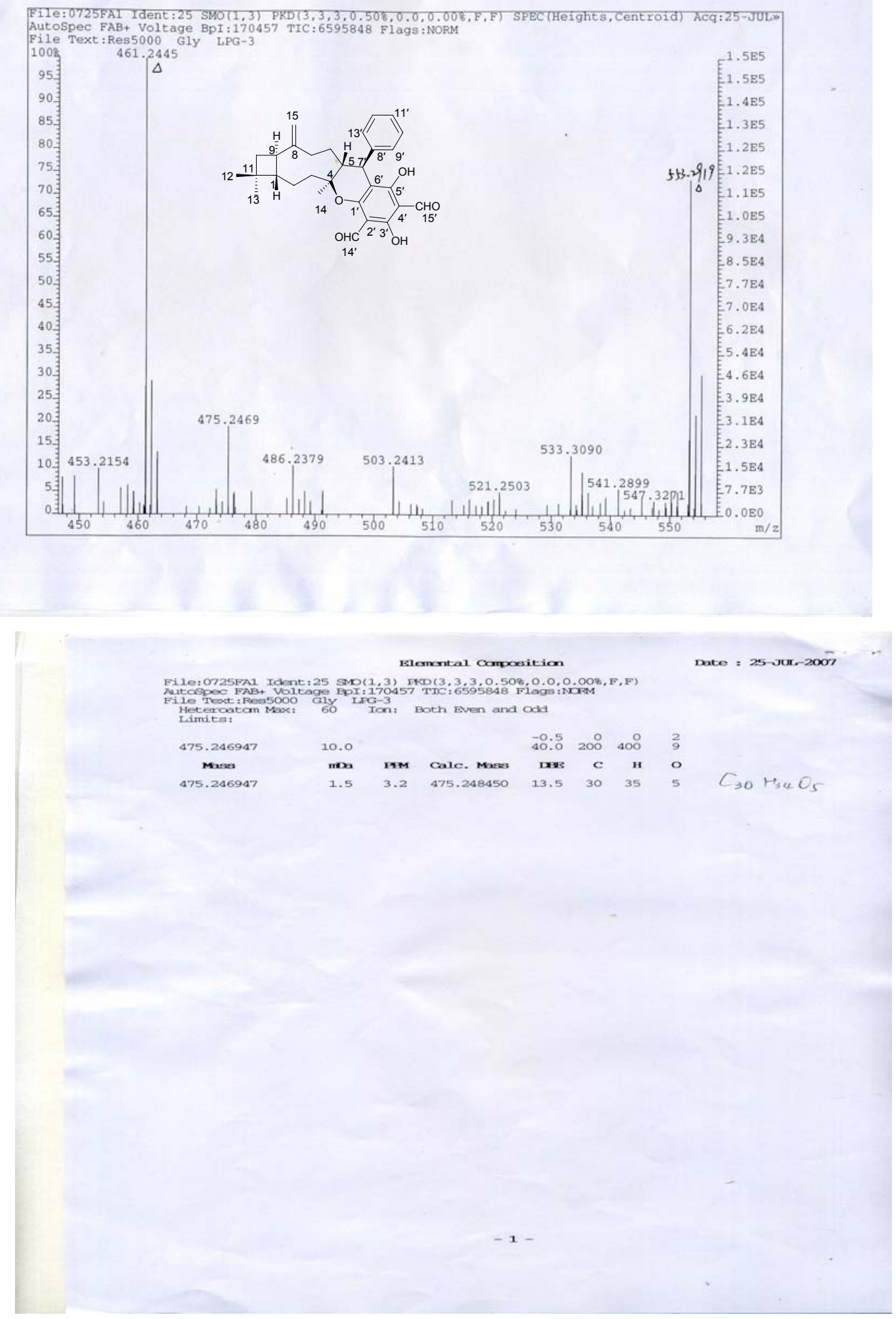

Date $=25-304-2007$ 
Figure S9. HRFAB-MS spectrum of fragment peak

at $m / z 271$ of Guajadial (1)

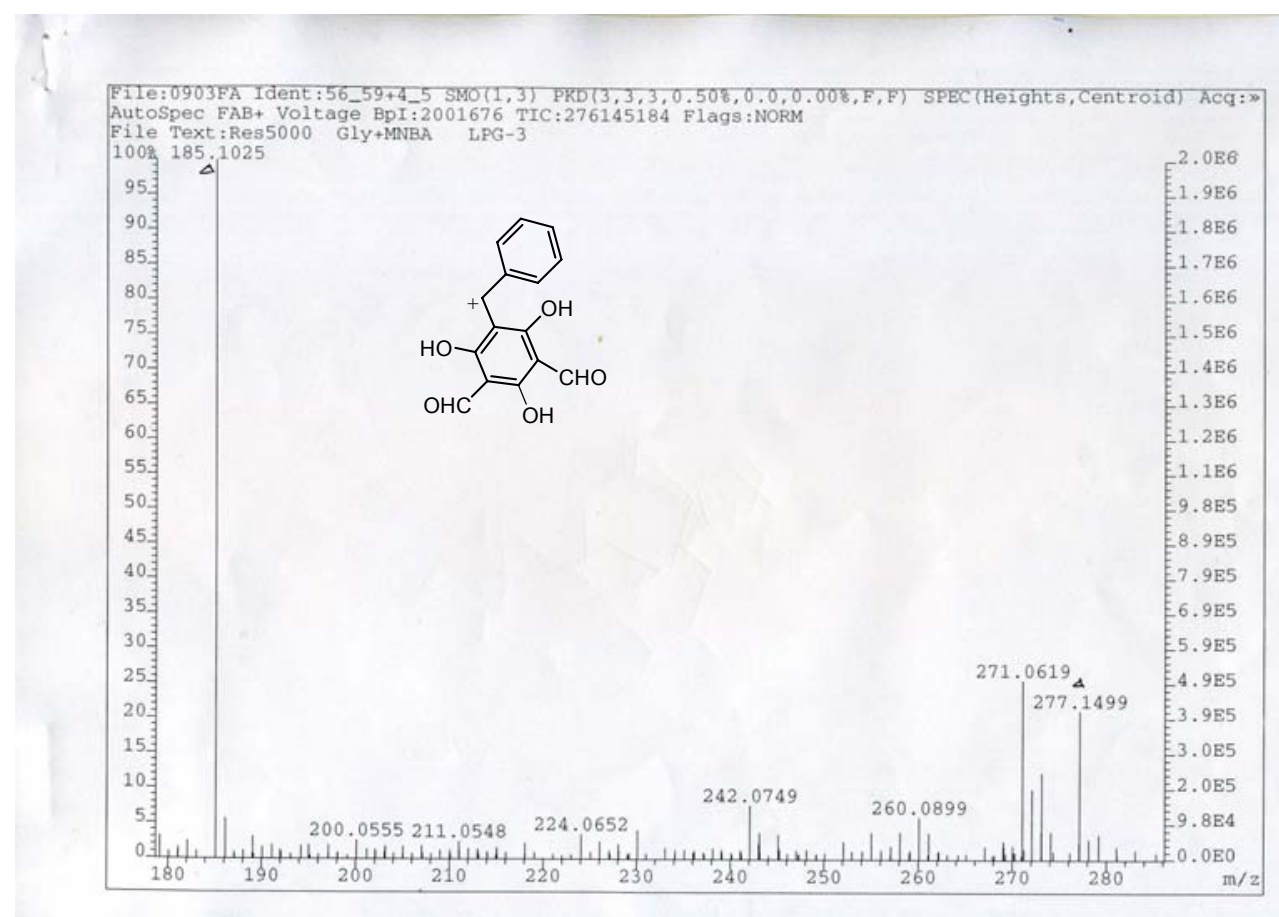

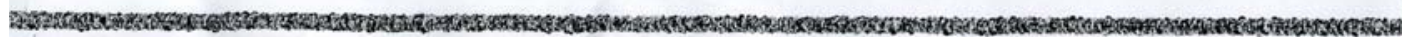

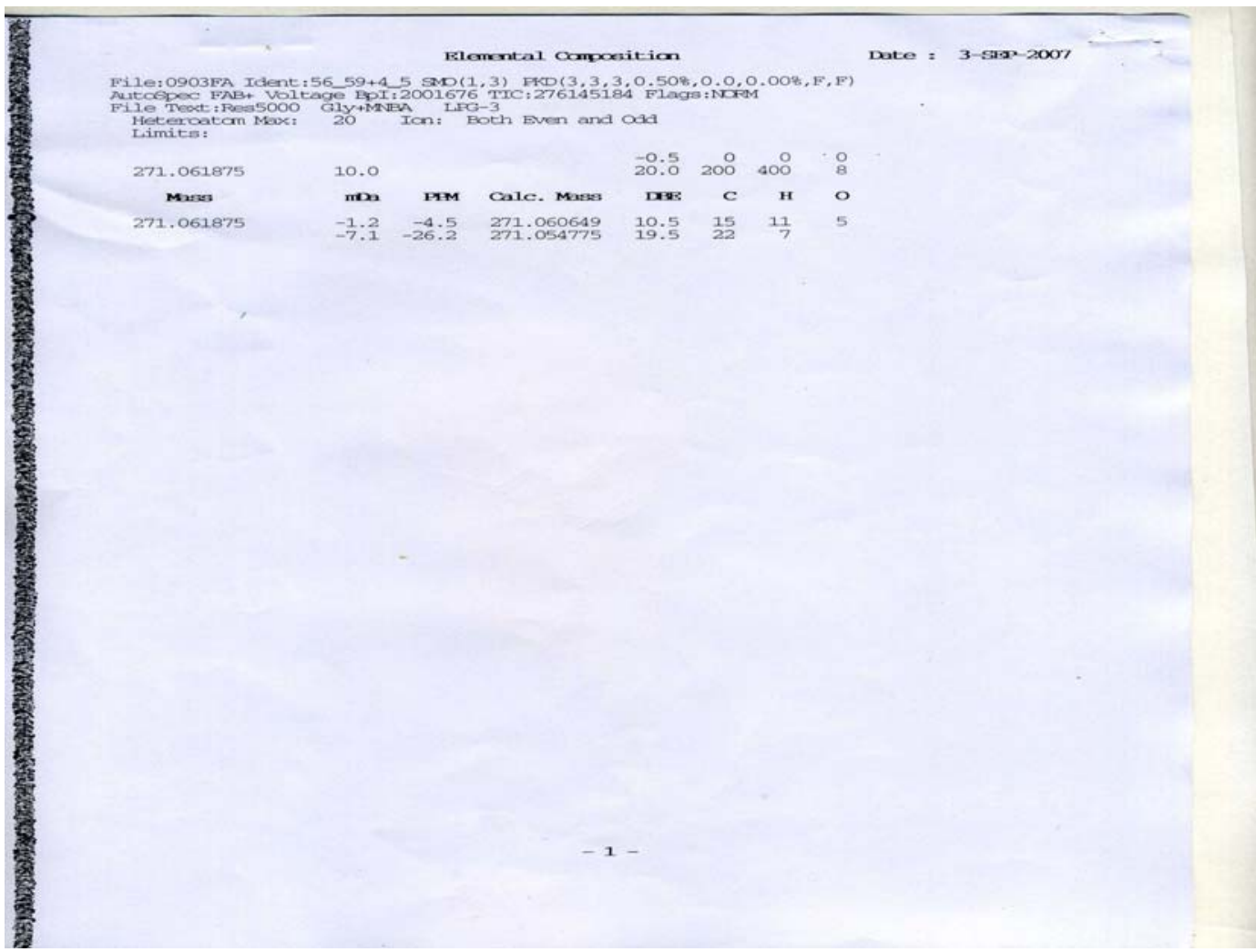


Figure S10. IR (KBr) spectrum of Guajadial (1)

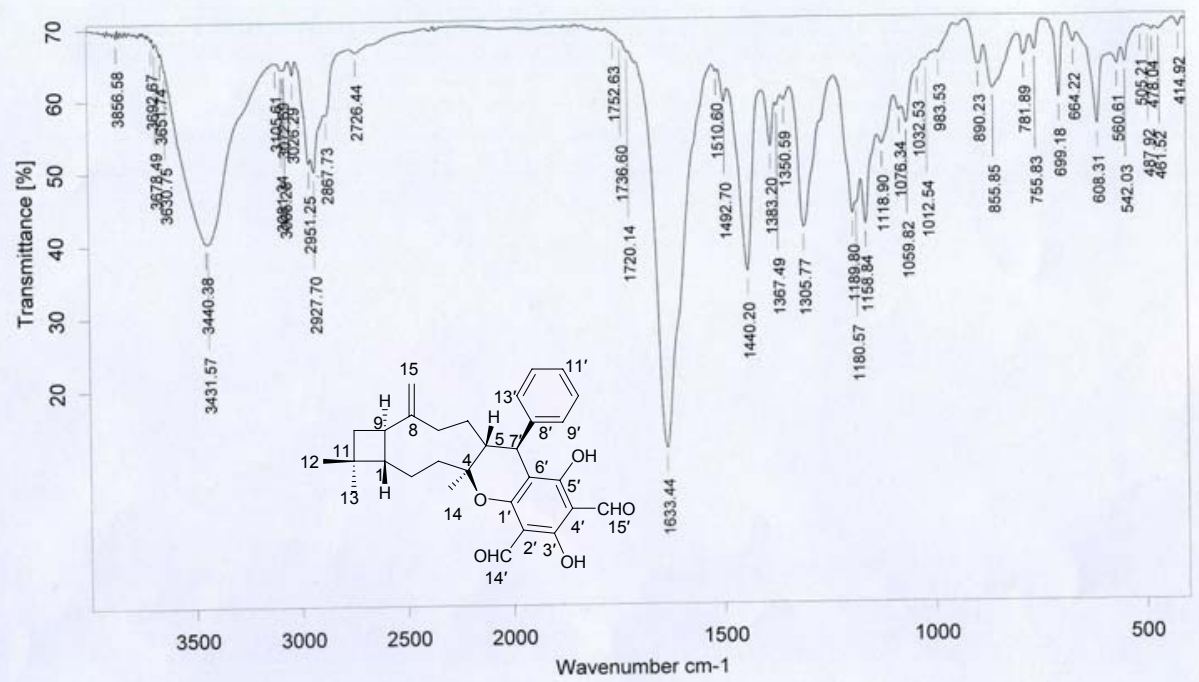

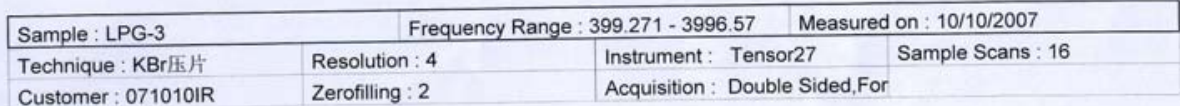

Figure S11. UV(MeOH) spectrum of Guajadial (1)

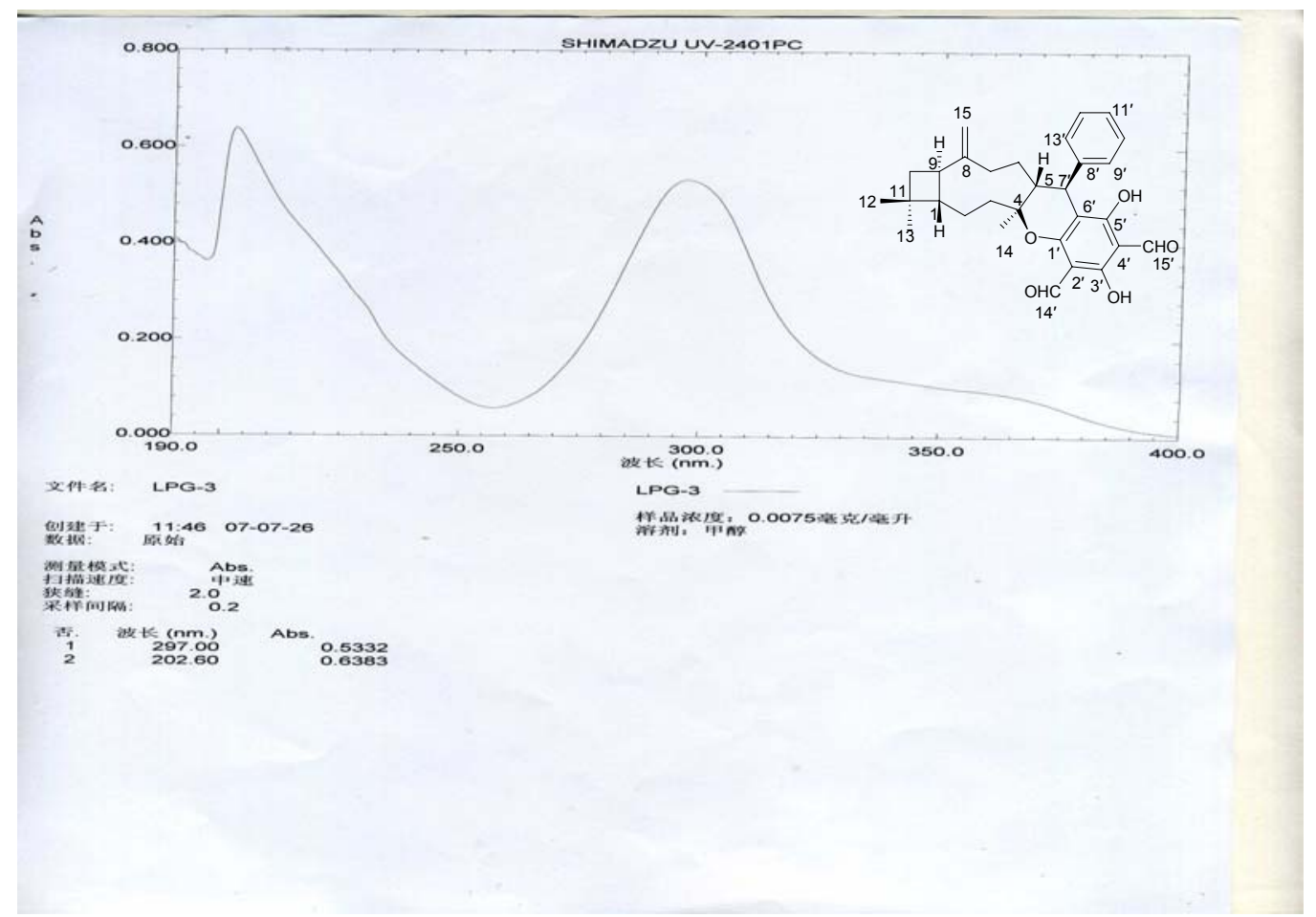


Figure S12. ${ }^{1} \mathrm{H}$ NMR spectrum of Guajadial (1, $500 \mathrm{MHz}, \mathbf{C}_{5} \mathbf{D}_{5} \mathbf{N}$ and $\left.\mathrm{CD}_{3} \mathrm{COCD}_{3}\right)$

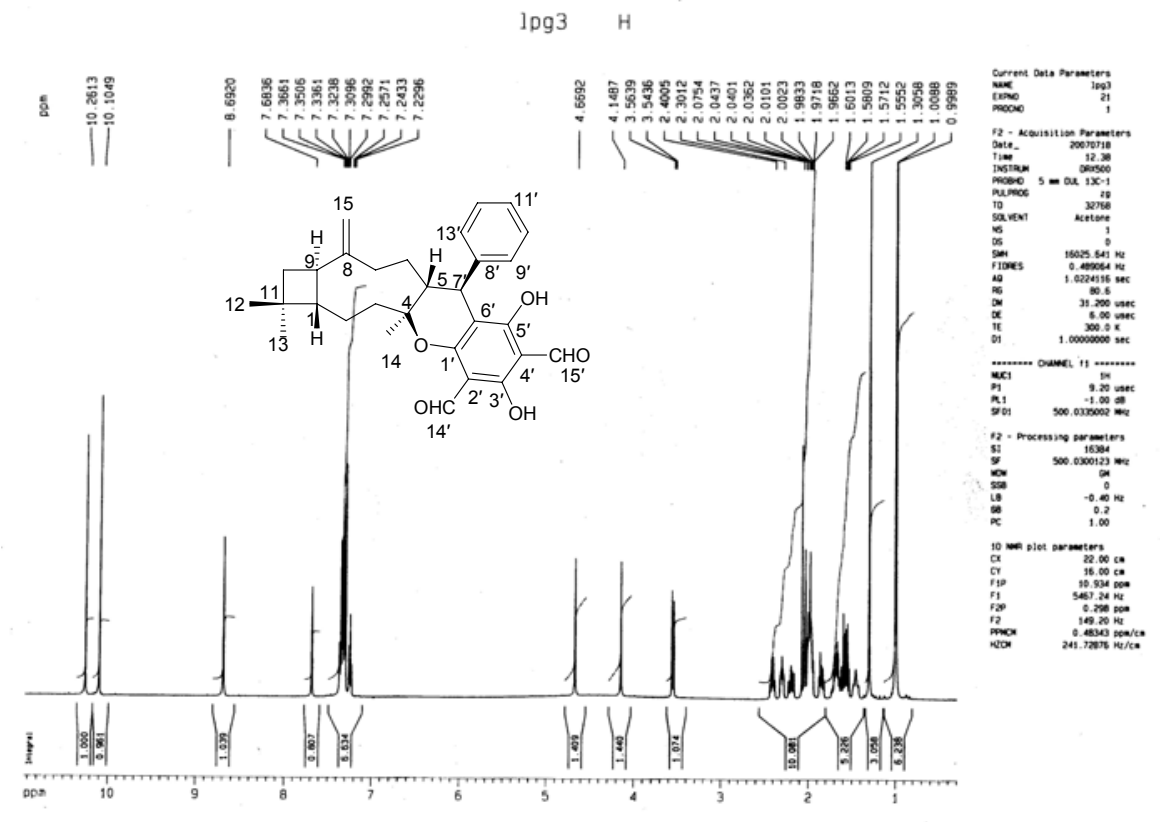

Figure S13. ${ }^{13} \mathrm{C}$ NMR spectrum of Guajadial $\left(\mathbf{1}, \mathbf{1 2 5} \mathbf{M H z}, \mathbf{C}_{5} \mathbf{D}_{5} \mathbf{N}\right.$ and $\left.\mathrm{CD}_{3} \mathrm{COCD}_{3}\right)$
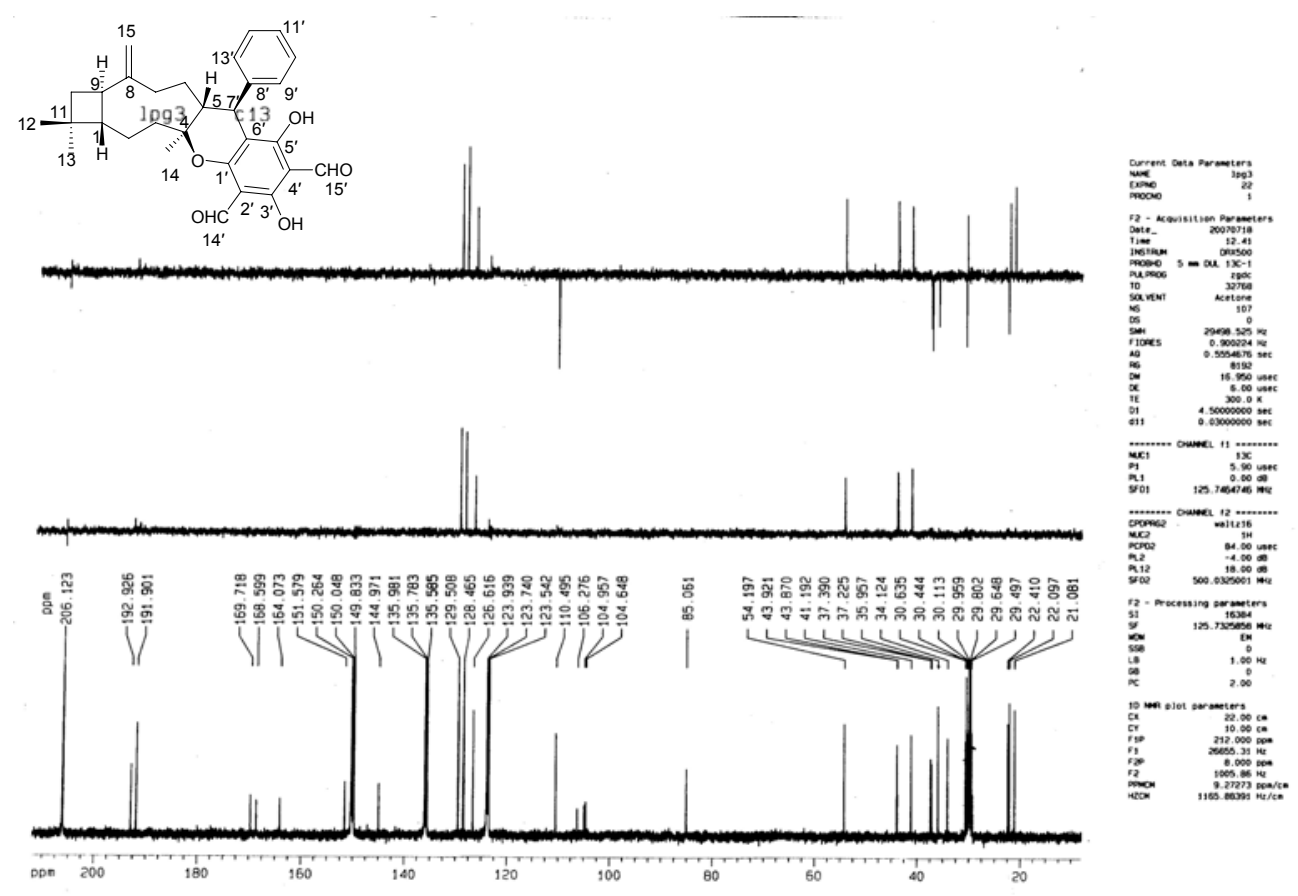
Figure S14. HSQC spectrum of Guajadial $\left(1, \mathbf{C}_{5} \mathbf{D}_{5} \mathbf{N}\right.$ and $\left.\mathbf{C D}_{3} \mathbf{C O C D}_{3}\right)$
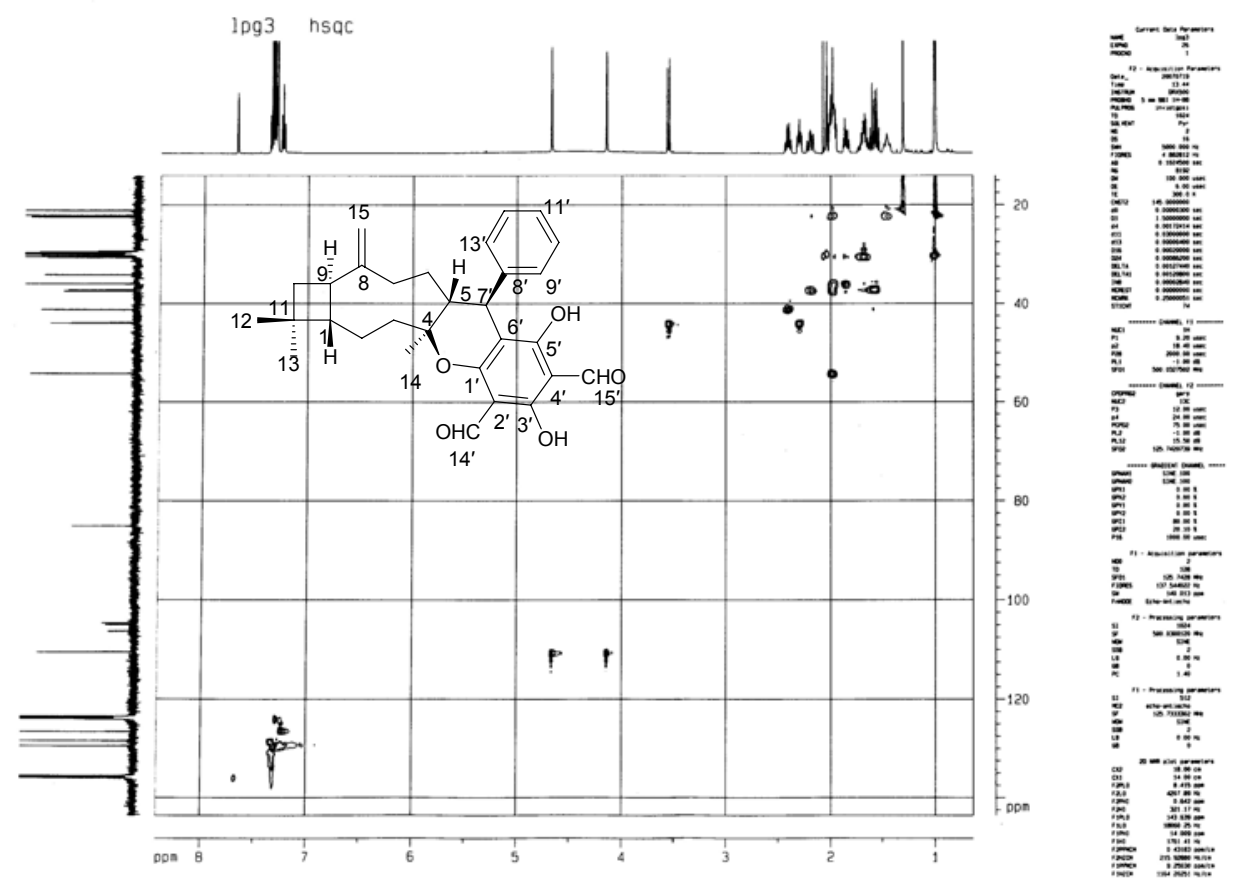

Figure S15. HMBC spectrum of Guajadial $\left(1, \mathbf{C}_{5} \mathbf{D}_{5} \mathbf{N}\right.$ and $\left.\mathrm{CD}_{3} \mathrm{COCD}_{3}\right)$
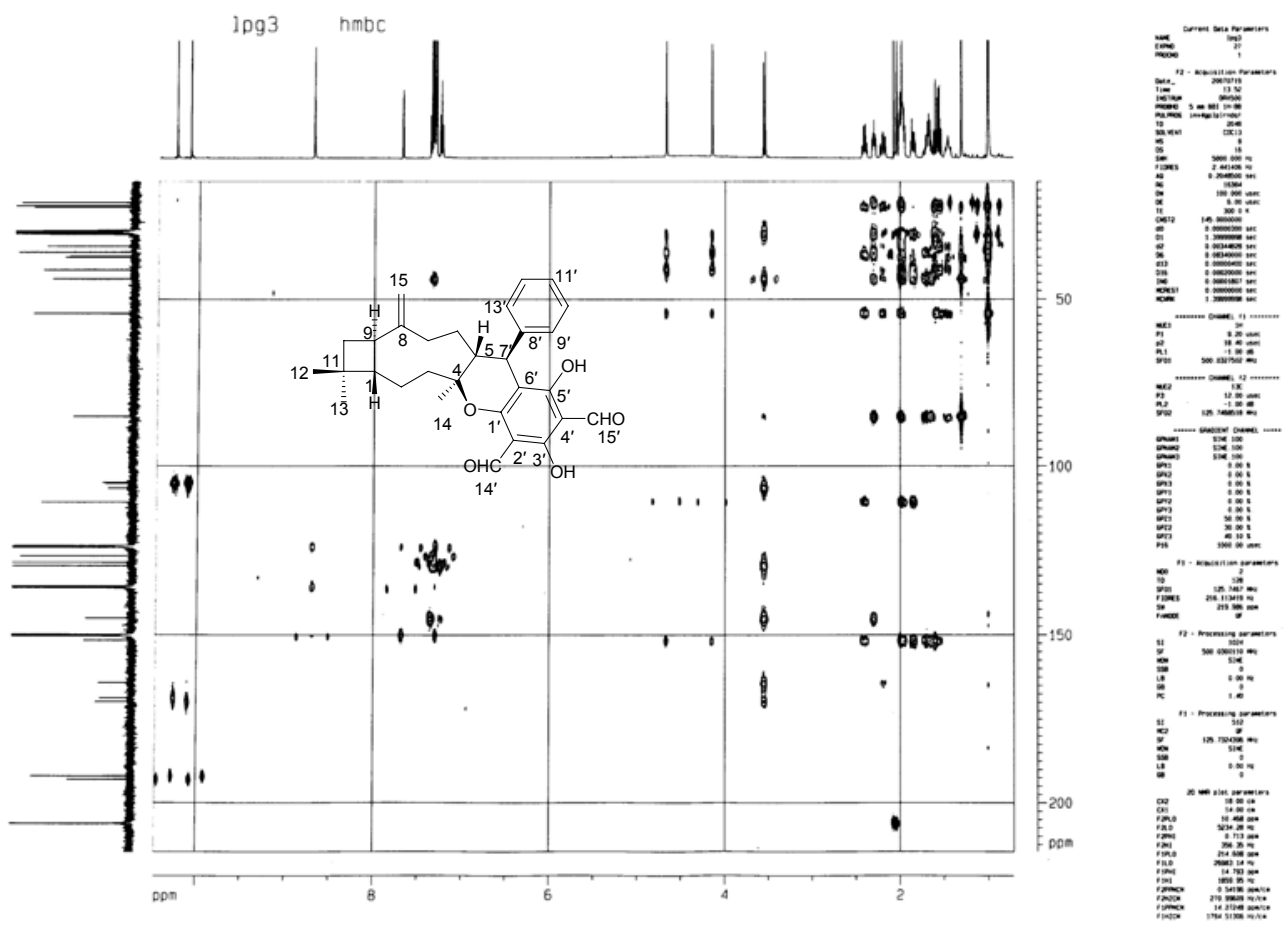
FigureS16. ${ }^{1} \mathrm{H}-{ }^{1} \mathrm{H}$ COSY spectrum of Guajadial $\left(\mathbf{1}, \mathbf{C}_{5} \mathbf{D}_{5} \mathbf{N}\right.$ and $\left.\mathrm{CD}_{3} \mathrm{COCD}_{3}\right)$
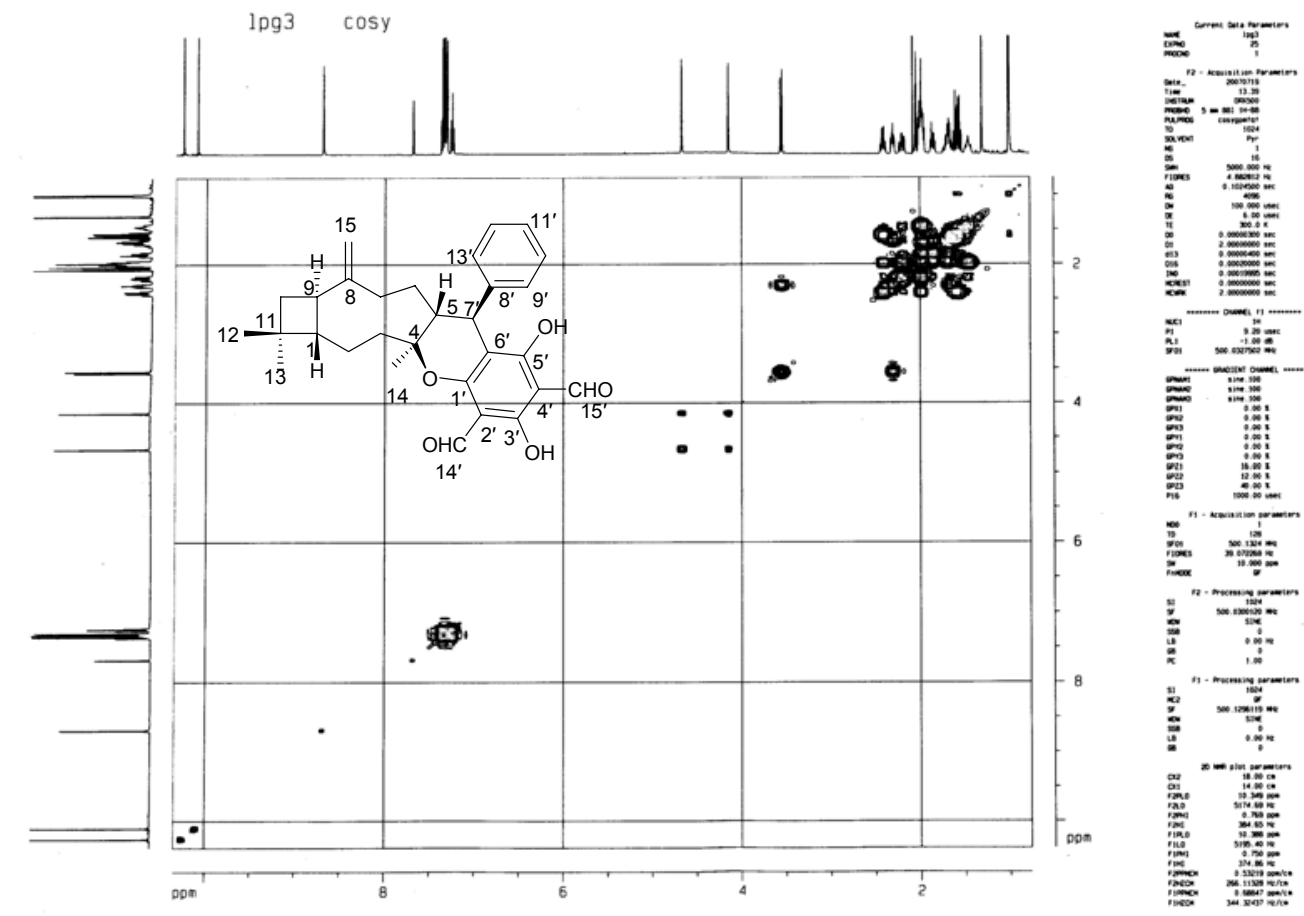

Figure S17. ROESY spectrum of Guajadial $\left(1, \mathbf{C}_{5} \mathbf{D}_{5} \mathbf{N}\right.$ and $\left.\mathbf{C D}_{3} \mathbf{C O C D}_{3}\right)$

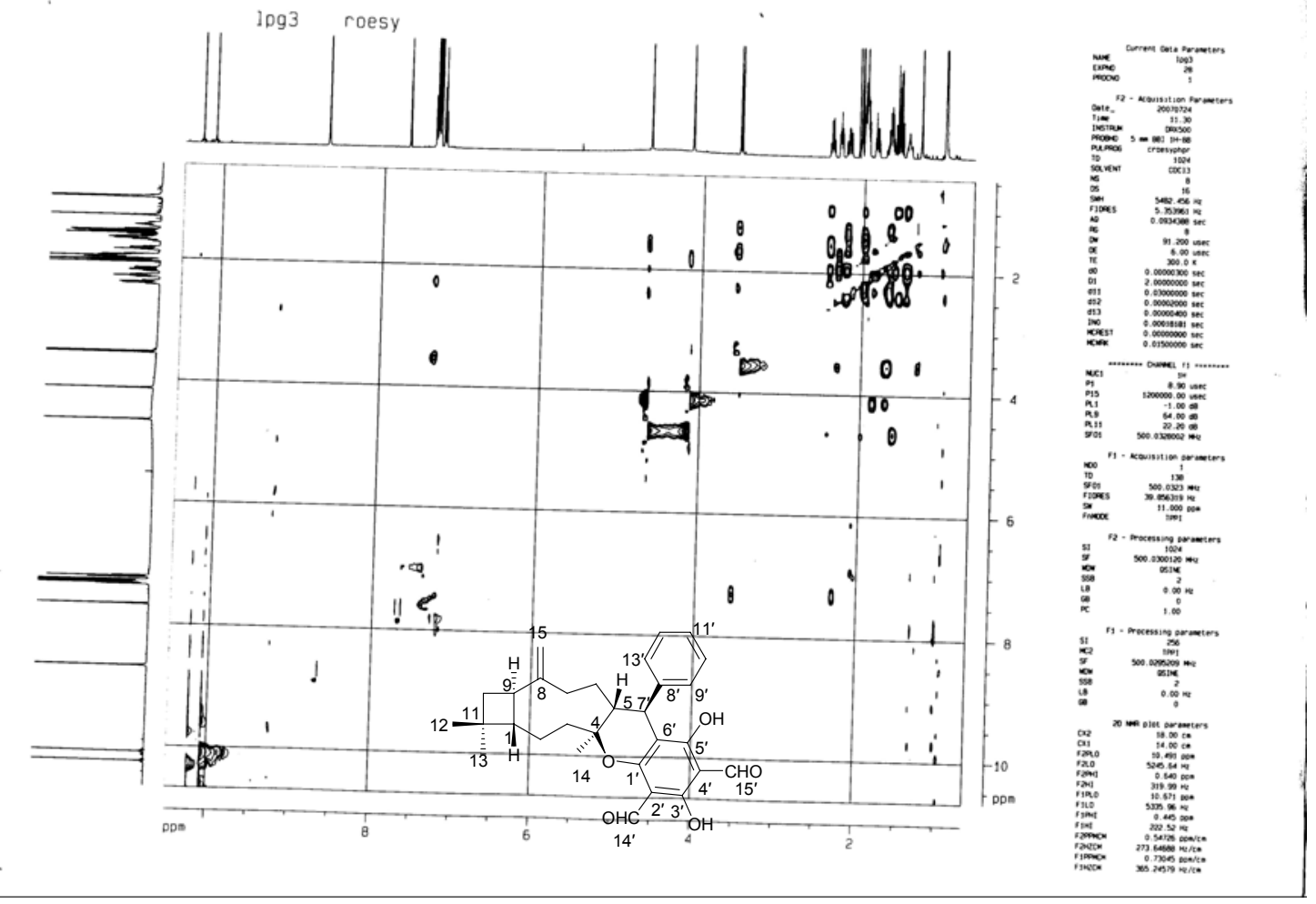

